Geometry $\&$ Topology

Volume 9 (2005) 971-990

Published: 29 May 2005

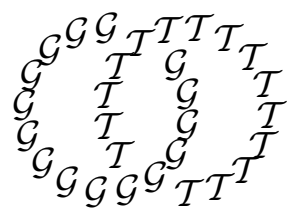

\title{
End reductions, fundamental groups, and covering spaces of irreducible open 3-manifolds
}

\author{
ROBERT MYERS \\ Department of Mathematics, Oklahoma State University \\ Stillwater, OK 74078, USA \\ Email: myersr@math.okstate.edu \\ URL: http://www.math.okstate.edu/ myersr/
}

\begin{abstract}
Suppose $M$ is a connected, open, orientable, irreducible 3-manifold which is not homeomorphic to $\mathbb{R}^{3}$. Given a compact 3 -manifold $J$ in $M$ which satisfies certain conditions, Brin and Thickstun have associated to it an open neighborhood $V$ called an end reduction of $M$ at $J$. It has some useful properties which allow one to extend to $M$ various results known to hold for the more restrictive class of eventually end irreducible open 3-manifolds.

In this paper we explore the relationship of $V$ and $M$ with regard to their fundamental groups and their covering spaces. In particular we give conditions under which the inclusion induced homomorphism on fundamental groups is an isomorphism. We also show that if $M$ has universal covering space homeomorphic to $\mathbb{R}^{3}$, then so does $V$.

This work was motivated by a conjecture of Freedman (later disproved by Freedman and Gabai) on knots in $M$ which are covered by a standard set of lines in $\mathbb{R}^{3}$.
\end{abstract}

AMS Classification numbers Primary: 57M10

Secondary: $57 \mathrm{~N} 10,57 \mathrm{M} 27$

Keywords: 3-manifold, end reduction, covering space

Proposed: David Gabai

Received: 14 July 2004

Seconded: Walter Neumann, Cameron Gordon

Revised: 18 May 2005

(c) Geometry ${ }^{\mathcal{S}} \mathcal{T}$ Topology Publications 


\section{Introduction}

The Marden Conjecture states that an open hyperbolic 3-manifold with finitely generated fundamental group is almost compact, ie is homeomorphic to the interior of a compact 3-manifold. Independent proofs of this conjecture have recently been given by Ian Agol [1] and by Danny Calegari and David Gabai [5]. These proofs use techniques which are mostly geometric. In 9] Mike Freedman proposed the following topological conjecture which he proved implies the Marden Conjecture.

Conjecture 1.1 (Freedman Conjecture) Let $M$ be a connected, orientable open 3-manifold and $p: \widetilde{M} \rightarrow M$ its universal covering map. Let $\gamma$ be a knot in $M$. If

(1) $\pi_{1}(M)$ is finitely generated,

(2) $\widetilde{M}$ is homeomorphic to $\mathbb{R}^{3}$, and

(3) $p^{-1}(\gamma)$ is a standard set of lines,

then $\pi_{1}(M-\gamma)$ is finitely generated.

By a standard set of lines we mean a subset $L$ of $\mathbb{R}^{3}$ such that $\left(\mathbb{R}^{3}, L\right)$ is homeomorphic to $\left(\mathbb{R}^{2}, X\right) \times \mathbb{R}$, where $X$ is a countably infinite closed discrete subset of $\mathbb{R}^{2}$.

In fact Freedman showed that the following special case of his conjecture implies the Marden Conjecture.

Conjecture 1.2 (Special Freedman Conjecture) Let $M$ and $\gamma$ be as above. Assume that, in addition,

(4) $\pi_{1}(M)$ is a free product of two non-trivial groups,

(5) $[\gamma] \in \pi_{1}(M)$ is algebraically disk busting, and

(6) $\langle\gamma\rangle=0 \in H_{1}\left(M, \mathbb{Z}_{2}\right)$.

Then $\pi_{1}(M-\gamma)$ is finitely generated.

An element $g$ of a group $G$ is algebraically disk busting if it is not conjugate into a proper free factor in any free factorization of $G$. For example, let $G$ be the free group on the set $\{a, b\}$, and let $g=a^{2} b^{2}$. If $g$ were conjugate into a proper free factor of $G$, then the quotient of $G$ by the normal closure of $g$ 
would have the form $\mathbb{Z} * \mathbb{Z}_{n}$, which contradicts the fact that this quotient is the fundamental group of the Klein bottle.

This paper was originally part of a program for attacking Conjecture 1.2. After it was written Freedman and Gabai discovered a counterexample [8 to this conjecture, and so that program is now defunct. (We note that they had earlier found a counterexample to Conjecture 1.1.)

The idea of the program was to split the problem into two parts: proving the conjecture for the case in which $M$ is "end irreducible rel $\gamma$ ", and reducing the conjecture to this special case. (See the next section for definitions.) This paper is concerned with the reduction to the special case. The FreedmanGabai counterexample is end irreducible rel $\gamma$, and so is a counterexample to the special case.

The device for trying to do the reduction is an "end reduction of $M$ at $\gamma$ ". This is a certain connected open subset $V$ of $M$ which is end irreducible rel $\gamma$ and has certain other nice properties. (The theory of end reductions was developed in more general contexts by Brin and Thickstun [2, 3], who were in turn inspired by earlier work of Brown and Feustel [4.)

The inclusion induced map $\pi_{1}(V) \rightarrow \pi_{1}(M)$ is injective. Assuming the context of the Special Freedman Conjecture we prove in Theorem 5.1 that this homomorphism is also surjective, so $p^{-1}(V)$ is connected; in Theorem 6.1 we prove that it is homeomorphic to $\mathbb{R}^{3}$ and in Theorem 8.1 that $p^{-1}(\gamma)$ is a standard set of lines in $p^{-1}(V)$.

The reduction would have worked as follows. Let $V$ be an end reduction of $M$ at $\gamma$. Since $\pi_{1}(V)$ is isomorphic to $\pi_{1}(M)$ it is finitely generated and $\gamma$ is algebraically disk busting in $\pi_{1}(V)$. Then $p^{-1}(V)$ is homeomorphic to $\mathbb{R}^{3}$ and $p^{-1}(\gamma)$ is a standard set of lines in $p^{-1}(V)$. If the conjecture were true for $V$ and $\gamma$, then $\pi_{1}(V-\gamma)$ would be finitely generated. The reduction would have been complete if the following has an affirmative answer.

Question 1.3 Does $\pi_{1}(V-\gamma)$ finitely generated imply that $\pi_{1}(M-\gamma)$ is finitely generated?

The paper is organized as follows. Sections 2 and 3 give an exposition of portions of Brin and Thickstun's theory of end reductions. Section 4 proves the incidental result that if $\pi_{1}(M)$ is finitely generated and indecomposable, then $V$ is either simply connected or $\pi_{1}$-surjective. Section 5 proves that if $\pi_{1}(M)$ is finitely generated and decomposable and $V$ is an end reduction of $M$ at an algebraically disk busting knot $\gamma$, then $V$ is $\pi_{1}$-surjective. Section 6 proves that if $V$ is any 
end reduction of any irreducible, orientable open 3-manifold $M$ whose universal covering space is homeomorphic to $\mathbb{R}^{3}$, then each component of $p^{-1}(V)$ is homeomorphic to $\mathbb{R}^{3}$. Section 7 proves some lemmas about trivial $k$ component tangles which are then used in section 8 to prove that if $p^{-1}(\gamma)$ is a standard set of lines in $\widetilde{M} \approx \mathbb{R}^{3}$, then the intersection of each component of $p^{-1}(V)$ with $p^{-1}(\gamma)$ is a standard set of lines.

Agol noticed that the proof that $\pi_{1}(V)$ and $\pi_{1}(M)$ are isomorphic when $\gamma$ is algebraically disk busting works in a more general context and used this in his proof of the Marden Conjecture. Section 9 shows how to modify the proof of Theorem 5.1 to obtain a result which includes the situation considered by Agol. We note that Calegari and Gabai also used end reductions in their proof.

This research was partially supported by NSF Grant DMS-0072429.

\section{End reductions}

In general we follow 11] or [12 for basic 3-manifold terminology. When $X$ is a submanifold of $Y$ we denote the topological interior of $X$ by $\operatorname{Int} X$ and the manifold interior of $X$ by int $X$. We say that $X$ is proper in $Y$ if $X \cap C$ is compact for each compact $C \subseteq Y$ and that it is properly embedded in $Y$ if $X \cap \partial Y=\partial X$. Two compact properly embedded surfaces $F$ and $G$ in a 3 -manifold are in minimal general position if they are in general position and for all surfaces isotopic to $F$ and in general position with respect to $G$ the intersection of $F$ and $G$ has the smallest number of components.

Throughout the paper $M$ will be a connected, orientable, irreducible, open 3manifold which is not homeomorphic to $\mathbb{R}^{3}$. A sequence $\left\{C_{n}\right\}_{n \geq 0}$ of compact, connected 3-manifolds $C_{n}$ in $M$ such that $C_{n} \subseteq \operatorname{int} C_{n+1}$ and $M-\operatorname{int} C_{n}$ has no compact components is called a quasi-exhaustion in $M$. If $\cup C_{n}=M$, then it is called an exhaustion for $M$.

In this section and the next we presents some basic material about Brin and Thickstun's theory of end reductions. See 2, 3, for more details. We note that they work in a more general context and give somewhat different definitions of the terms that follow. In our context the definition of end reduction is equivalent to theirs.

A compact, connected $3-$ manifold $J$ in $M$ is regular in $M$ if $M-J$ is irreducible and has no component with compact closure. Since $M$ is irreducible the first condition is equivalent to the statement that $J$ does not lie in a 3-ball in $M$. A quasi-exhaustion $\left\{C_{n}\right\}$ in $M$ is regular if each $C_{n}$ is regular in $M$. 
Let $J$ be a regular 3-manifold in $M$, and let $V$ be an open subset of $M$ which contains $J$. We say that $V$ is end irreducible rel $J$ in $M$ if there is a regular quasi-exhaustion $\left\{C_{n}\right\}$ in $M$ such that $V=\cup_{n \geq 0} C_{n}, J=C_{0}$, and $\partial C_{n}$ is incompressible in $M-\operatorname{int} J$ for all $n>0$. In the case that $V=M$ we say that $M$ is end irreducible rel $J$; we say that $M$ is eventually end irreducible if it is end irreducible rel $J$ for some $J$.

$V$ has the engulfing property rel $J$ in $M$ if whenever $N$ is regular in $M$, $J \subseteq \operatorname{int} N$, and $\partial N$ is incompressible in $M-J$, then $V$ is ambient isotopic rel $J$ to $V^{\prime}$ such that $N \subseteq V^{\prime}$. (Brin and Thickstun's "weak engulfing" property requires the isotopy to be fixed off a compact subset of $M-J$, but this can be achieved by using the covering isotopy theorem [6, 7].)

$V$ is an end reduction of $M$ at $J$ if $V$ is end irreducible rel $J$ in $M, V$ has the engulfing property rel $J$ in $M$, and no component of $M-V$ is compact. (Note that $\mathbb{R}^{3}$ contains no regular 3 -manifolds and hence has no end reductions, so we will henceforth not mention the hypothesis that $M$ is not homeomorphic to $\mathbb{R}^{3}$.)

Theorem 2.1 (Brin-Thickstun) Given a regular 3-manifold $J$ in $M$, an end reduction $V$ of $M$ at $J$ exists and is unique up to non-ambient isotopy rel $J$ in $M$.

Proof This follows from Theorems 2.1 and 2.3 of [2].

We will need to understand the construction of $V$. Here is a brief sketch. We begin with a regular exhaustion $\left\{C_{n}\right\}_{n \geq 0}$ of $M$ with $C_{0}=J$. Set $C_{0}^{*}=C_{0}$. If $\partial C_{1}$ is incompressible in $M-J$ set $C_{1}^{*}=C_{1}$. Otherwise we "completely compress" $\partial C_{1}$ in $M-C_{0}^{*}$ to obtain $C_{1}^{*}$. This is done by constructing a sequence of compact 3 -manifolds $K_{0}, \ldots, K_{p}$ with $K_{0}=C_{1}$ and $K_{p}=C_{1}^{*}$ as follows. $K_{i+1}$ is obtained from $K_{i}$ by one of the following two operations. One may cut off a 1-handle from $K_{i}$ which misses $C_{0}$ and whose co-core meets $\partial K_{i}$ in an essential simple closed curve. (This is just compressing $\partial K_{i}$ "to the inside".) One may add a 2-handle to $K_{i}$ in $M-C_{0}$ whose core meets $\partial K_{i}$ in an essential simple closed curve. (This is just compressing $\partial K_{i}$ "to the outside".) Moreover the cutting and attaching occurs along disjoint annuli in $\partial C_{1}$. We may assume that $C_{1}^{*} \subseteq \operatorname{int} C_{2}$. If $\partial C_{2}$ is incompressible in $M-J$ we set $C_{2}^{*}=C_{2}$. Otherwise we completely compress $\partial C_{2}$ in $M-C_{1}^{*}$ to get $C_{2}^{*}$. We continue in this fashion to construct a sequence $\left\{C_{n}^{*}\right\}_{n \geq 0}$. We let $V^{*}=\cup_{n \geq 0} C_{n}^{*}$ and then let $V$ be the component of $V^{*}$ containing $J . V^{*}$ is called a constructed end reduction of $M$ at $J$. We will call $V$ a standard end reduction of $M$ at $J$. 
It will be convenient to arrange for $V$ to be obtained solely through cutting 1-handles. The following result is in Brown and Feustel [4] and has antecedents in work of McMillan [13, 14, 15.

Lemma 2.2 (Thick Exhaustion Lemma (Brown-Feustel)) There is an exhaustion $\left\{\widehat{C}_{n}\right\}$ for $M$ such that $C_{n} \subseteq \widehat{C}_{n}$ and $C_{n}^{*}$ can be obtained from $\widehat{C}_{n}$ by cutting 1-handles.

Proof Suppose that in the process of completely compressing $\partial C_{n}$ in $M-C_{n-1}$ we first cut some 1 -handles and then add some 2-handles. We may assume that the cores of these handles are in general position. If the intersection is non-empty, then the 2-handles cut the 1-handles into a collection of shorter 1-handles. Thus one could obtain the same 3-manifold by first adding the 2-handles and then cutting a possibly greater number of 1-handles.

It follows that we can add all the 2-handles at once to obtain a $3-$ manifold $\widehat{C}_{n}$ from which we then cut 1 -handles to obtain $C_{n}^{*}$.

From now on we assume that our standard end reduction $V$ has been obtained by cutting 1 -handles.

It is clear that a standard end reduction $V$ is end irreducible rel $J$ in $M$.

Suppose $N$ is regular in $M, J \subseteq \operatorname{int} N$, and $\partial N$ is incompressible in $M-J$. Then $N \subseteq C_{n}$ for some $n$. We isotop $\partial N$ off the co-cores of the 1 -handles which are cut to obtain $C_{n}^{*}$. Hence $N$ is now contained in the component of $C_{n}^{*}$ which contains $J$ and hence is contained in $V$. Running the isotopy backwards establishes the engulfing property.

Suppose we give our cut 1 -handles product structures of the form $D \times[a, b]$. We may arrange things so that if $H$ and $H^{\prime}$ are 1-handles cut from $C_{n}$ and $C_{m}$, respectively, with $m>n$, then $H \cap H^{\prime}$ is either empty or consists of a finite number of 1-handles each of which has induced product structures from $H$ and from $H^{\prime}$. From this it follows that if $x \in C_{n} \cap\left(M-V^{*}\right)$, then $x$ lies in a properly embedded disk in $C_{n}$ which is in $M-V^{*}$. Also, if $x \in C_{n} \cap\left(V^{*}-V\right)$, then $x$ lies in a connected 3-manifold in $C_{n}$ which meets $\partial C_{n}$. It follows that $M-V$ has no compact component.

Another consequence of the 1-handles having compatible product structures is the widely noted but seemingly unrecorded observation, first made by Brin and Thickstun, that $M-V^{*}$ is a lamination of $M$ each leaf of which is a plane.

Now suppose that $J$ and $K$ are regular 3 -manifolds in $M$ such that $J \subseteq \operatorname{int} K$ and $\partial K$ is incompressible in $M-J$. Let $V$ be an end reduction of $M$ at $J$ 
such that $K \subseteq V$. In Theorem 2.2 of [2] Brin and Thickstun prove that if $L$ is regular in $M, K \subseteq \operatorname{int} L$, and $\partial L$ is incompressible in $M-K$, then $V$ is ambient isotopic rel $K$ to $V^{\prime}$ such that $L \subseteq V^{\prime}$. This is called the strong engulfing property. It follows from this property that $V$ is an end reduction of $M$ at $K$; see Corollary 2.2.1 of [2].

One can now prove the uniqueness of $V$ up to non-ambient isotopy rel $J$ by using the strong engulfing property to inductively isotop the elements of properly chosen exhaustions $\left\{V_{n}\right\}$ for $V$ and $\left\{V_{n}^{\prime}\right\}$ for another end reduction $V^{\prime}$ of $M$ at $J$ so that $V_{n}^{\prime} \subseteq V_{n} \subseteq V_{n+1}^{\prime}$ for all $n$. See Theorem 2.3 of [2].

Suppose $V$ is an end reduction of $M$ at $J$ and $h_{t}: V \rightarrow M, t \in[0,1]$, is a non-ambient isotopy with $h_{0}$ the inclusion map and $h_{t}(x)=x$ for all $x \in J$. By Lemma 2.5 of [2] $h_{1}(V)$ is an end reduction of $M$ at $J$. It is clear that $h_{1}(V)$ is end irreducible rel $J$ in $M$ and that $h_{1}(V)$ has the engulfing property rel $J$ in $M$. To see that $M-h_{1}(V)$ has no compact component we proceed as follows. (The author thanks Tom Thickstun for providing the following argument.) Let $\widehat{M}$ be the Freudenthal compactification of $M$. (See [10] or section 1.3 of [2].) The points of $\widehat{M}-M$ are the ends $E(M)$ of $M$. Since $M-V$ has no compact components each component of $\widehat{M}-V$ contains an end of $M$. It suffices to show that each component of $\widehat{M}-h_{1}(V)$ contains an end of $M$. Let $\left\{V_{n}\right\}$ be an exhaustion for $V$ such that each $V_{n}$ is regular in $M$. Thus each component of $\widehat{M}-V_{n}$ contains an end of $M$. By the covering isotopy theorem [6, 17] there is an ambient isotopy $g_{n, t}: \widehat{M} \rightarrow \widehat{M}$ rel $\widehat{M}-M$ such that $g_{n, t}(x)=h_{t}(x)$ for all $x \in V_{n}$. Therefore each component of $\widehat{M}-h_{1}\left(V_{n}\right)$ contains an end of $M$. Let $X$ be a component of $\widehat{M}-h_{1}(V)$. Then $X=\cap X_{i}$, where $X_{i}$ is a component of $\widehat{M}-h_{1}\left(V_{i}\right)$. Thus $X_{i}$ contains some $e_{i} \in E(M)$. We may assume that $e_{i} \rightarrow e \in \widehat{M}$. Since $E(M)$ is closed in $\widehat{M}$ and $X$ is the nested intersection of the $X_{i}$ we have that $e \in E(M) \cap X$. Thus $M-h_{1}(V)$ has no compact component.

We now define a regular knot in $M$ to be a simple closed curve $\gamma$ in $M$ which is not contained in a 3 -ball in $M$. In this case a regular neighborhood $J$ of $\gamma$ in $M$ is a regular $3-$ manifold in $M$, and we define an end reduction of $M$ at $\gamma$ to be an end reduction of $M$ at $J$. There is no loss of generality in working with end reductions at regular knots since every end reduction of $M$ at $J$ is also an end reduction at a regular knot in $M$. (See [16].) 


\section{$3 \quad \pi_{1}$-injectivity}

The following result is in Proposition 1.4 of [3]. We include a proof here to make our exposition more self contained.

Theorem 3.1 (Brin-Thickstun) Let $M$ be a connected, orientable, irreducible, open 3-manifold. Let $J$ be a regular 3-manifold in $M$ and $V$ an end reduction of $M$ at $J$. Then the inclusion induced homomorphism $\pi_{1}(V) \rightarrow$ $\pi_{1}(M)$ is injective.

Proof Since $V$ is unique up to non-ambient isotopy rel $J$ we may assume that $V$ is a standard end reduction associated to an exhaustion $\left\{C_{n}\right\}$ for $M$. By Lemma 2.2 we may assume that $C_{n}^{*}$ is obtained from $C_{n}$ by cutting 1 -handles. Now suppose that $\alpha$ is a loop in $V$ which bounds a singular disk in $M$. Thus we have a map $f: \Delta \rightarrow M$, where $\Delta$ is a disk and $f(\partial \Delta)=\alpha$.

We may assume that $\alpha \subseteq C_{1}^{*}$ and $f(\Delta) \subseteq C_{1}$. Let $D$ be the co-core of the first 1-handle which is cut from $C_{1}$ in the process of obtaining $C_{1}^{*}$. Put $f$ in general position with respect to $D$. Then $f^{-1}(D)$ consists of simple closed curves in int $\Delta$. We redefine $f$ in a neighborhood of the outermost disks among these curves so that $f^{-1}(D)=\emptyset$.

We then apply this procedure to the next 1 -handle which is cut and continue until we have that $f(\Delta) \subseteq C_{1}^{*}$ and hence $f(\Delta) \subseteq V$.

\section{$4 \quad \pi_{1}$-surjectivity: the indecomposable case}

In general, end reductions need not be $\pi_{1}$-surjective. In this section and the next we restrict to the case that $\pi_{1}(M)$ is finitely generated and place further restrictions which guarantee $\pi_{1}$-surjectivity.

Recall that the Scott compact core theorem [17] asserts that if $M$ is a connected non-compact 3-manifold with $\pi_{1}(M)$ finitely generated, then there is a compact, connected $3-$ manifold $N$ in int $M$ (a compact core) such that the inclusion induced homomorphism $\pi_{1}(N) \rightarrow \pi_{1}(M)$ is an isomorphism. Since we will be assuming that $M$ is irreducible and $\pi_{1}(M)$ is non-trivial we may cap off any $2-$ sphere components of $\partial N$ with 3 -balls in $M-\operatorname{int} N$ and hence may assume that $\partial N$ contains no 2 -spheres.

Note that if we are in a situation in which we wish to show that $\pi_{1}(V) \rightarrow \pi_{1}(M)$ is an isomorphism it suffices to show that there is a compact core $N$ such that $N \subseteq V$. 
Theorem 4.1 Let $M$ be a connected, orientable, irreducible, open 3-manifold. Let $J$ be a regular 3-manifold in $M$ and $V$ an end reduction of $M$ at $J$. Suppose that $\pi_{1}(V)$ is non-trivial and that $\pi_{1}(M)$ is finitely generated, non-cyclic, and indecomposable. Then the inclusion induced homomorphism $\pi_{1}(V) \rightarrow$ $\pi_{1}(M)$ is an isomorphism.

Proof We may assume that $V$ is a standard end reduction obtained from an exhaustion $\left\{C_{n}\right\}$ for $M$. We may further assume that $C_{n}^{*}$ is obtained from $C_{n}$ by cutting 1 -handles and that a compact core $N$ lies in $\operatorname{int} C_{1}$. Since $\pi_{1}(M)$ is indecomposable and non-cyclic we have that $\partial N$ is incompressible in $M$.

Let $D$ be the co-core of the first 1 -handle of $C_{1}$ which is cut. Isotop $N$ in int $C_{1}$ so that $\partial N$ is in general position with respect to $D$ and meets it in a minimal number of components. Each of these components bounds a disk on $\partial N$. Let $\Delta$ be an innermost such disk. Then $\partial \Delta=\partial \Delta^{\prime}$ for a disk $\Delta^{\prime}$ on $D$. The 2-sphere $\Delta \cup \Delta^{\prime}$ bounds a 3 -ball $B$ in $M$. Since $C_{1}$ is regular it is irreducible, and so $B$ lies in $\operatorname{int} C_{1}$. One may then isotop $\Delta$ across $B$ past $\Delta^{\prime}$ to remove at least one component of $D \cap \partial N$, contradicting minimality. Therefore $D \cap \partial N=\emptyset$.

In a similar fashion we see that $N$ meets none of the co-cores of the 1-handles which are cut to get $C_{1}^{*}$. Thus $N$ lies in $C_{1}^{*}$ and hence in $V^{*}$.

Suppose $N$ does not lie in $V$. Let $\alpha$ be a loop in $V$. Then $\alpha$ is freely homotopic in $M$ to a loop in $N$. We may assume that $\alpha$ and the image of the homotopy lie in $C_{2}$. We may further assume that $\alpha$ and $N$ lie in different components of $C_{2}^{*}$. It follows that the homotopy can be cut off on the co-core of some 1-handle which is cut to obtain $C_{2}^{*}$, and thus $\alpha$ is homotopically trivial. Hence $\pi_{1}(V)$ is trivial, contradicting our hypothesis.

Thus $N$ lies in $V$, which completes the proof.

\section{$5 \quad \pi_{1}$-surjectivity: the algebraically disk busting case}

Theorem 5.1 Let $M$ be a connected, orientable, irreducible, open 3-manifold. Let $\gamma$ be a regular knot in $M$ and $V$ an end reduction of $M$ at $\gamma$. Suppose that $\pi_{1}(M)$ is finitely generated and decomposable and that $\gamma$ is algebraically disk busting. Then the inclusion induced homomorphism $\pi_{1}(V) \rightarrow \pi_{1}(M)$ is an isomorphism. 
Proof As before we may assume that $V$ is a standard end reduction obtained from the exhaustion $\left\{C_{n}\right\}$ by cutting 1 -handles. Let $N$ be a compact core for $M$. We may assume that $N \subseteq \operatorname{int} C_{1}$ and that $\gamma$ is freely homotopic in $\operatorname{int} C_{1}$ to a knot $\gamma^{\prime} \subseteq \operatorname{int} N$.

Let $D$ be the co-core of the first 1-handle which is cut from $C_{1}$. Let $C_{1}^{\prime}$ be the resulting 3-manifold. Note that $\gamma \cap D=\emptyset$.

$N$ is either a cube with handles or consists of a finite number of 3-manifolds with boundaries which are incompressible in $M$ to which 1-handles have been added. It follows that $N$ can be chosen so that $N \cap D$ consists of essential disks in $N$. Assume that among all choices of $N$ the number of these disks is minimal.

Suppose $N \cap D \neq \emptyset$. Isotop $\gamma^{\prime}$ in $\operatorname{int} C_{1}$ so that $\gamma^{\prime} \subseteq \operatorname{int} N, \gamma^{\prime}$ is in general position with respect to $D$ and $\gamma^{\prime} \cap D$ has a minimal number of points.

If $\gamma^{\prime} \cap D=\emptyset$, then some essential disk in $N$ misses $\gamma^{\prime}$. It follows that $\gamma^{\prime}$ is conjugate into a proper free factor of $\pi_{1}(N)$ and hence that $\gamma$ is not algebraically disk busting in $\pi_{1}(M)$, a contradiction.

Thus $\gamma^{\prime} \cap D \neq \emptyset$. We will show that, contrary to our assumptions, there is a compact core $N^{\prime}$ in $C_{1}$ which meets $D$ in fewer disks than $N$ does.

We have a map $f: S^{1} \times[0,1] \rightarrow C_{1}$ with $f\left(S^{1} \times\{0\}\right)=\gamma$ and $f\left(S^{1} \times\{1\}\right)=\gamma^{\prime}$. Put $f$ in general position with respect to $D$. Then $f^{-1}(D)$ consists of simple closed curves and arcs with boundary in $S^{1} \times\{1\}$. Since $\gamma^{\prime} \cap D \neq \emptyset$ there must be a component of the latter type. Its union with some arc on $S^{1} \times\{1\}$ bounds a disk in $S^{1} \times[0,1]$. We may assume that the interior of this disk is disjoint from $f^{-1}(D)$. The disk provides a path homotopy in $C_{1}$ between an arc $\alpha$ in $\gamma^{\prime}$ and a path $\beta$ in $D$.

Case $1 \partial \beta$ lies in a single component $\Delta$ of $N \cap D$.

Then $\beta$ is homotopic rel $\partial \beta$ in $D$ to an $\operatorname{arc} \delta$ in $\Delta$ such that $\delta \cap \gamma^{\prime}=\partial \delta$. Let $\gamma^{\prime \prime}=\gamma^{\prime} \cup \delta-\alpha$. Isotop $\gamma^{\prime \prime}$ slightly so that $\delta$ is moved off $D$ and $\gamma^{\prime \prime} \cap D=$ $\left(\gamma^{\prime} \cap D\right)-\partial \alpha$. Thus $\gamma^{\prime \prime}$ meets $D$ in fewer points than did $\gamma^{\prime}$, a contradiction.

Case $2 \partial \beta$ lies in two different components $\Delta_{0}$ and $\Delta_{1}$ of $N \cap D$.

Then $\beta$ is homotopic rel $\partial \beta$ in $D$ to an $\operatorname{arc} \delta$ in $D$ which meets each $\Delta_{i}$ in an $\operatorname{arc} \delta_{i}$ and is otherwise disjoint from $N$. Push $\delta$ slightly off $D$ and add a 1-handle to $N$ whose core is $\delta \cap\left(C_{1}-\operatorname{Int} N\right)$. Then cut a 1 -handle from $N$ whose co-core is $\Delta_{1}$. Call the result $N^{\prime}$. Since $\alpha$ is path homotopic to $\beta$ the core of the cut 1 -handle is path homotopic to an arc in $N^{\prime}$, from which it 
follows that $N^{\prime}$ is a compact core for $M$. Although it no longer contains $\gamma^{\prime}$ it meets $D$ in one fewer disk, contradicting our assumption on $N$.

Thus we must have that $N \cap D=\emptyset$. We repeat the argument with $C_{1}^{\prime}$ in place of $C_{1}$ and continue until we get that $N \subseteq C_{1}^{*}$. As in Theorem 4.1 we get that $N \subseteq V$, and we are done.

\section{$6 \quad \widetilde{V}$ is homeomorphic to $\mathbb{R}^{3}$}

Theorem 6.1 Let $M$ be a connected, orientable, irreducible, open 3-manifold. Let $J$ be a regular 3-manifold in $M$ and $V$ an end reduction of $M$ at $J$. Let $p: \widetilde{M} \rightarrow M$ be the universal covering map. Let $\widetilde{V}$ be a component of $p^{-1}(V)$. If $\widetilde{M}$ is homeomorphic to $\mathbb{R}^{3}$, then so is $\widetilde{V}$.

Proof Since $V$ is $\pi_{1}$-injective in $M$ we have that $\widetilde{V}$ is simply connected. It suffices to show that for every compact, connected subset $A$ of $\widetilde{V}$ there exists a 3-ball in $\widetilde{V}$ whose interior contains $A$.

We may assume that $V$ is the end reduction obtained from an exhaustion $\left\{C_{n}\right\}$ for $M$ by cutting 1 -handles.

Since $\widetilde{M}$ is homeomorphic to $\mathbb{R}^{3}$, there is a 3 -ball $B$ in $\widetilde{M}$ whose interior contains $A$. We may assume that $(p(B), p(A)) \subseteq\left(C_{1}, C_{1}^{*} \cap V\right)$.

Suppose $D$ is the co-core of the first 1 -handle of $C_{1}$ which is cut. So $D$ compresses $\partial C_{1}$ in $C_{1}-J$. Let $C_{1}^{\prime}$ be the resulting 3 -manifold. Then $p^{-1}(D)$ is a disjoint union of disks $\widetilde{D}_{i}$ in $p^{-1}\left(C_{1}\right)$. Since the group of covering translations is properly discontinuous only finitely many of these disks meet $B$. None of them meets $A$. Put $\partial B$ in general position with respect to $p^{-1}(D)$.

Assume that for some $i$ we have $\widetilde{D}_{i} \cap B \neq \emptyset$. Let $\Delta$ be an innermost disk on $\widetilde{D}_{i}$, ie $\Delta \cap \partial B=\partial \Delta$.

Suppose $\Delta$ lies in $B$. Then it splits $B$ into two 3 -balls one of which, say $B^{\# \text {, }}$ contains $A$. Then $B^{\#}$ can be isotoped to remove $\partial \Delta$ from the intersection.

Now suppose $\Delta$ lies in $\widetilde{M}-\operatorname{int} B$. Attaching a $2-$ handle to $B$ with core $\Delta$ gives a 3 -manifold $Q$ with $\partial Q$ a disjoint union of two 2 -spheres. One of them bounds a $3-$ ball $B^{\#}$ in $\widetilde{M}$ which contains $Q$. Then $\partial B^{\#}$ has at least one fewer intersection with $p^{-1}(D)$ than did $\partial B$.

Continuing in this fashion we get a 3 -ball $B^{\prime}$ in $\widetilde{M}$ such that $B^{\prime} \cap p^{-1}(D)=\emptyset$. So $A \subseteq \operatorname{int} B^{\prime}$ and $B^{\prime} \subseteq \operatorname{int} p^{-1}\left(C_{1}^{\prime}\right)$.

We then proceed to the cutting of the next 1-handle. Eventually we get a 3-ball $B^{*}$ in $\widetilde{M}$ such that $A \subseteq \operatorname{int} B^{*}$ and $B^{*} \subseteq p^{-1}\left(C_{1}^{*} \cap V\right) \subseteq \widetilde{V}$. 


\section{$7 \quad$ Standard embeddings of trivial $k$-tangles}

A $k$-tangle is a pair $(B, \tau)$, where $B$ is a 3 -ball and $\tau$ is a union of $k$ disjoint properly embedded $\operatorname{arcs} \tau_{i}$ in $B .(B, \tau)$ and $\left(B^{\prime}, \tau^{\prime}\right)$ have the same tangle type if there is a homeomorphism of pairs $h:(B, \tau) \rightarrow\left(B^{\prime}, \tau^{\prime}\right)$. We say that $(B, \tau)$ is trivial if it has the same tangle type as $(D, P) \times[0,1]$, where $D$ is a disk and $P$ is a set of $k$ points in int $D$.

A shell is a $3-$ manifold $\Sigma$ which is homeomorphic to $S^{2} \times[0,1]$. Let $\beta$ be a union of $k$ disjoint properly embedded $\operatorname{arcs} \beta_{i}$ in $\Sigma$ such that each $\beta_{i}$ joins $S^{2} \times\{0\}$ to $S^{2} \times\{1\} . \quad(\Sigma, \beta)$ is a $k$-braid if there is a homeomorphism $g:(\Sigma, \beta) \rightarrow$ $\left(S^{2}, P\right) \times[0,1]$, where $P$ is a set of $k$ points in $S^{2}$.

Lemma $7.1(1)(B, \tau)$ is a trivial $k$-tangle if and only if there is a disjoint union $F$ of $k$ disks $F_{i}$ in $B$ such that for $1 \leq i \leq k$ one has that $F_{i} \cap \tau=\tau_{i}$ and $F_{i} \cap \partial B=\partial F_{i}-\operatorname{int} \tau_{i}$.

(2) $(\Sigma, \beta)$ is a $k$-braid if and only if for some (and hence any) $\beta_{j}$ its exterior is a 3 -ball $X$ such that either $k=1$ or $k>1$ and $\beta-\beta_{j}$ is a trivial $(k-1)$ tangle in $X$ for which there exist disks $F_{i}$ as in (1) each of which has connected intersection with the annulus $\partial X-\operatorname{int}(X \cap \partial \Sigma)$.

Proof The proof is left as an exercise.

Lemma 7.2 (1) Suppose $(B, \tau)$ is a trivial $k$-tangle and $G$ is a finite disjoint union of properly embedded disks in $B$ which misses $\tau$. Let $B^{\prime}$ be the closure of a component of $B-G$ such that $B^{\prime} \cap \tau \neq \emptyset$. Then $\left(B^{\prime}, B^{\prime} \cap \tau\right)$ is a trivial $k^{\prime}$-tangle for some $k^{\prime} \leq k$.

(2) Suppose $(\Sigma, \beta)$ is a $k$-braid and $G$ is a finite disjoint union of properly embedded disks in $S^{2} \times(0,1]$ which misses $\beta$. Let $\Sigma^{\prime}$ be the closure of the component of $\Sigma-G$ which contains $S^{2} \times\{0\}$. Then $\left(\Sigma^{\prime}, \beta\right)$ is a $k$-braid.

Proof (1) Let $F$ be as in Lemma 7.1(1). Put $F$ in minimal general position with respect to $G$. Since the exterior of $\tau$ in $B$ is a cube with handles it is irreducible; it follows that $G \cap F$ has no simple closed curve components. Let $\tau^{\prime}=\tau \cap B^{\prime}$. For each component $\tau_{i}$ of $\tau^{\prime}$ let $F_{i}^{\prime}$ be the closure of the component of $F_{i}-\left(F_{i} \cap G\right)$ which contains $\tau_{i}$. It then follows from Lemma 7.1 (1) that $\left(B^{\prime}, \tau^{\prime}\right)$ is trivial.

(2) Let $\beta_{j}, X$, and $F$ be as in Lemma 7.1(2). Again putting $F$ in minimal

general position with respect to $G$, no component of $G \cap F$ is a simple closed 
curve. We have that $\beta$ lies in $\Sigma^{\prime}$. For each component $\beta_{i}$ of $\beta-\beta_{j}$ let $F_{i}^{\prime}$ be the closure of the component of $F_{i}-\left(F_{i} \cap G\right)$ which contains $\beta_{i}$. Then $F_{i}^{\prime}$ lies in $X^{\prime}=X \cap \Sigma^{\prime}$ and has connected intersection with $\partial X^{\prime}-\operatorname{int}\left(X \cap \partial \Sigma^{\prime}\right)$. Thus by Lemma $7.1(2)\left(\Sigma^{\prime}, \beta\right)$ is a $k$-braid.

Let $B$ and $\widehat{B}$ be 3 -balls, $\tau$ a $k$-tangle in $B$, and $\widehat{\tau}$ a $\widehat{k}$-tangle in $\widehat{B}$. We say that $(B, \tau)$ is standardly embedded in $(\widehat{B}, \widehat{\tau})$ if (i) $B \subseteq \operatorname{int} \widehat{B}$, (ii) $\widehat{\tau} \cap B=\tau$, and (iii) there is a finite disjoint union $\widehat{\mathcal{D}}$ of properly embedded disks in $\widehat{B}-B$ such that $\widehat{\mathcal{D}} \cap \widehat{\tau}=\emptyset$ and $\widehat{\mathcal{D}}$ splits $\widehat{B}-\operatorname{int} B$ into a shell $\widehat{\Sigma}$ which meets $\widehat{\tau}$ in a $2 k$-braid and a disjoint union $\widehat{\mathcal{B}}$ of 3 -balls $\widehat{B}_{j}$ each of which meets $\widehat{\tau}$ in a trivial $k_{j}$-tangle $\left(k_{j}\right.$ depending on the 3 -ball $\widehat{B}_{j}$ ).

Lemma 7.3 Suppose $(B, \tau)$ is standardly embedded in $(\widehat{B}, \widehat{\tau})$. Then

(1) one may choose $\widehat{\mathcal{D}}$ (and hence $\widehat{\mathcal{B}}$ ) to be connected,

(2) for each component $\widehat{\tau}_{i}$ of $\widehat{\tau}$ one has that $\widehat{\tau}_{i} \cap B$ is connected,

(3) if $(B, \tau)$ is trivial then so is $(\widehat{B}, \widehat{\tau})$, and

(4) if $(\widehat{B}, \widehat{\tau})$ is standardly embedded in $(\widetilde{B}, \widetilde{\tau})$, then $(B, \tau)$ is standardly embedded in $(\widetilde{B}, \widetilde{\tau})$.

Proof (1) and (3) follow from the fact that if $\left(B^{\prime}, \tau^{\prime}\right)$ is a trivial $k^{\prime}$-tangle and $\left(B^{\prime \prime}, \tau^{\prime \prime}\right)$ is a trivial $k^{\prime \prime}$-tangle such that $B^{\prime} \cap B^{\prime \prime}$ is a disk which is disjoint from $\tau^{\prime} \cup \tau^{\prime \prime}$, then $\left(B^{\prime} \cup B^{\prime \prime}, \tau^{\prime} \cup \tau^{\prime \prime}\right)$ is a trivial $\left(k^{\prime}+k^{\prime \prime}\right)$-tangle.

(3) follows from the fact that $\widehat{\tau} \cap \Sigma$ is a $2 k$-braid.

By (1) we may assume that $\widehat{\mathcal{D}}$ is connected and that there is a properly embedded disk $\widetilde{\mathcal{D}}$ in $\widetilde{B}-\widehat{B}$ which splits $\widetilde{B}-$ int $\widehat{B}$ into a shell $\widetilde{\Sigma}$ and a $3-$ ball $\widetilde{\mathcal{B}}$ such that $(\widetilde{\Sigma}, \widetilde{\Sigma} \cap \widetilde{\tau})$ is a $2 \widetilde{k}$-braid and $(\widetilde{\mathcal{B}}, \widetilde{\mathcal{B}} \cap \widetilde{\tau})$ is a trivial $(\widetilde{k}-\widehat{k})$-tangle. Let $\widetilde{A}=(\partial \widehat{\mathcal{D}}) \times[0,1]$ in the product structure on $\widetilde{\Sigma}$. Since $\widetilde{\tau}$ misses $\widetilde{\mathcal{D}}$ we may isotop $\widetilde{A}$ rel $\partial \widehat{\mathcal{D}}$ so that it misses $\widetilde{\mathcal{D}}$. Then $\widehat{\mathcal{D}} \cup \widetilde{A}$ splits $\widetilde{B}-\operatorname{int} B$ into a shell which meets $\widetilde{\tau}$ in a $2 k$-braid and a 3 -ball which meets $\widetilde{\tau}$ in a trivial $(\widetilde{k}-k)$-tangle. Thus we have $(4)$.

Lemma 7.4 Suppose $(B, \tau)$ is standardly embedded in $(\widehat{B}, \widehat{\tau})$. Let $\widehat{E}$ be a properly embedded disk in $\widehat{B}-B$ which is disjoint from $\widehat{\tau}$. Let $B^{\#}$ be the closure of the component of $\widehat{B}-\widehat{E}$ which contains $B$. Let $\tau^{\#}=\widehat{\tau} \cap B^{\#}$. Then $(B, \tau)$ is standardly embedded in $\left(B^{\#}, \tau^{\#}\right)$. 
Proof Since the definition of standardly embedded does not depend on the tangle type of $(B, \tau)$ we may assume that $(B, \tau)$ is trivial. By Lemma $7.3(1)$ we may assume that $\widehat{\mathcal{D}}$ is connected. Put it in minimal general position with respect to $\widehat{E}$. The exterior of $\widehat{\tau} \cap(\widehat{B}-\operatorname{int} B)$ in $\widehat{B}-\operatorname{int} B$ is a cube with handles and so is irreducible. It follows that $\widehat{\mathcal{D}} \cap \widehat{E}$ has no simple closed curve components.

Thus $\widehat{\mathcal{D}} \cap B^{\#}$ is a disjoint union $\mathcal{D}^{\#}$ of disks. These disks split $B^{\#}-\operatorname{int} B$ into a shell $\Sigma^{\#}$ and a disjoint union $\mathcal{B}^{\#}$ of 3 -balls. By deleting some of these disks we may assume that each of these 3 -balls meets $\tau^{\#}$. By Lemma 7.3 (3) we have that $(\widehat{B}, \widehat{\tau})$ is trivial, so by Lemma $7.2(1)\left(B^{\#}, \tau^{\#}\right)$ is trivial. A second application of Lemma 7.2 (1) shows that the intersection of $\tau^{\#}$ with each component of $\mathcal{B}^{\#}$ is a trivial tangle in that 3 -ball. By Lemma 7.3 (2) we have that $\left(\Sigma^{\#}, \Sigma^{\#} \cap \tau^{\#}\right)$ is a $2 k$-braid. Hence $(B, \tau)$ is standardly embedded in $\left(B^{\#}, \tau^{\#}\right)$.

Lemma 7.5 Let $(B, \tau),(\widehat{B}, \widehat{\tau})$, and $\left(B^{\#}, \tau^{\#}\right)$ be as in the previous lemma. Suppose $(\widehat{B}, \widehat{\tau})$ is standardly embedded in $(\widetilde{B}, \widetilde{\tau})$. Then $\left(B^{\#}, \tau^{\#}\right)$ is standardly embedded in $(\widetilde{B}, \widetilde{\tau})$.

Proof Recall that $B^{\#}$ is the closure of the component of $\widehat{B}-\widehat{E}$ which contains $B$. Since $(\widehat{B}, \widehat{\tau})$ is standardly embedded in $(\widetilde{B}, \widetilde{\tau})$ there is a properly embedded disk $\widetilde{\mathcal{D}}$ in $\widetilde{B}-\widehat{B}$ which splits $\widetilde{B}-\operatorname{int} \widehat{B}$ into a shell $\widetilde{\Sigma}$ and a $3-$ ball $\widetilde{\mathcal{C}}$.

Let $\widetilde{A}$ be the annulus $\partial \widehat{E} \times[0,1]$ in $\widetilde{\Sigma}=(\partial \widehat{B}) \times[0,1]$. Note that we may have $\widetilde{A} \cap \widetilde{\mathcal{D}} \neq \emptyset$. Since $\widetilde{\tau} \cap \widetilde{\mathcal{D}}=\emptyset$ we may isotop $\widetilde{A}$ in $\widetilde{\Sigma}-\widetilde{\tau}$ so that $\widetilde{A} \cap \widetilde{\mathcal{D}}=\emptyset$. We then deform the product structure on $\widetilde{\Sigma}$ so that we still have $\widetilde{A}=\partial \widehat{E} \times[0,1]$.

Now let $\widetilde{E}$ be a parallel copy of $\widehat{E} \cup \widetilde{A}$ in the component of $\widetilde{B}-(\widehat{E} \cup \widetilde{A})$ which does not contain $B$. We may assume that $\widetilde{E} \cap(\widetilde{\tau} \cup \widetilde{\mathcal{D}})=\emptyset$. Now $\widetilde{E}$ splits $\widetilde{B}-\operatorname{int} B^{\#}$ into a shell $\Sigma^{+}$and a 3 -ball $\mathcal{C}^{+}$.

Note that $\Sigma^{+}$is the union of the parallelism $(\widehat{E} \cup \widetilde{A}) \times[0,1]$ between $\widehat{E} \cup \widetilde{A}$ and $\widetilde{E}$ and $\left(\partial B^{\#}-\operatorname{int} \widehat{E}\right) \times[0,1]$, where the latter product structure is that of $\widetilde{\Sigma}$. It follows that $\left(\Sigma^{+}, \Sigma^{+} \cap \widetilde{\tau}\right)$ is a braid.

Let $\mathcal{C}_{0}^{+}$be the closure of the component of $\widetilde{B}-(\widetilde{E} \cup \widetilde{\mathcal{D}})$ such that $\partial \mathcal{C}_{0}^{+}$contains $\widetilde{E} \cup \widetilde{\mathcal{D}}$. Then $\mathcal{C}_{0}^{+} \cap \widehat{B}$ is a 3 -ball which contains $\widehat{\tau}-\tau^{\#}$. As in the proof of Lemma 7.4 we may assume that $(B, \tau)$ is trivial, and thus by Lemma 7.3 (3) so is $(\widehat{B}, \widehat{\tau})$, and thus by Lemma $7.2(1)$ so is $\left(\mathcal{C}_{0}^{+} \cap \widehat{B}, \widehat{\tau}-\tau^{\#}\right)$. Now $\mathcal{C}_{0}^{+}-\operatorname{Int} \widehat{B}$ is a 3 -ball whose intersection with $\widetilde{\tau}$ consists of product arcs in the product structure induced by that of $\widetilde{\Sigma}$. It follows that $\left(\mathcal{C}_{0}^{+}, \mathcal{C}_{0}^{+} \cap \widetilde{\tau}\right)$ is a trivial tangle. 
Since $(\widetilde{\mathcal{C}}, \widetilde{\mathcal{C}} \cap \widetilde{\tau})$ is trivial by the definition of standardly embedded, we have that $\left(\mathcal{C}_{0}^{+} \cup \widetilde{\mathcal{C}}, \widetilde{\tau}-\tau^{\#}\right)$ is trivial. Hence $\left(B^{\#}, \tau^{\#}\right)$ is standardly embedded in $(\widetilde{B}, \widetilde{\tau})$.

\section{$8 \tilde{V} \cap p^{-1}(\gamma)$ is standard in $\widetilde{V}$}

Recall that a proper 1 -manifold $L$ in $\mathbb{R}^{3}$ is a standard set of lines if there is a homeomorphism $H:\left(\mathbb{R}^{3}, L\right) \rightarrow\left(\mathbb{R}^{2}, X\right) \times \mathbb{R}$, where $X$ is a countably infinite closed discrete subset of $\mathbb{R}^{2}$.

Theorem 8.1 Let $M$ be a connected, orientable, irreducible open 3-manifold. Let $\gamma$ be a regular knot in $M$ and $V$ an end reduction of $M$ at $\gamma$. Let $p: \widetilde{M} \rightarrow M$ be the universal covering map. Suppose $\widetilde{M}$ is homeomorphic to $\mathbb{R}^{3}$ and $p^{-1}(\gamma)$ is a standard set of lines in $\widetilde{M}$. Then for each component $\widetilde{V}$ of $p^{-1}(V)$ we have that $\widetilde{V} \cap p^{-1}(\gamma)$ is a standard set of lines in $\widetilde{V}$.

Recall that by Theorem $6.1 \widetilde{V}$ is homeomorphic to $\mathbb{R}^{3}$. We will make use of the following lemma.

Lemma 8.2 $L$ is a standard set of lines in $\mathbb{R}^{3}$ if and only if there is an exhaustion $\left\{B_{n}\right\}_{n \geq 0}$ for $\mathbb{R}^{3}$ with each $B_{n}$ a 3-ball such that

(1) for each $n \geq 0 \quad B_{n} \cap L$ is a $k_{n}$-tangle $\tau^{n}$,

(2) $\left(B_{0}, \tau^{0}\right)$ is trivial, and

(3) for each $n \geq 0\left(B_{n}, \tau^{n}\right)$ is standardly embedded in $\left(B_{n+1}, \tau^{n+1}\right)$.

Proof Let $q: \mathbb{R}^{2} \times \mathbb{R} \rightarrow \mathbb{R}^{2}$ be projection onto the first factor.

First suppose that $L$ is standard.

Choose an exhaustion $\left\{E_{n}\right\}_{n \geq 0}$ of $\mathbb{R}^{2}$ such that each $E_{n}$ is a disk, $E_{0} \cap$ $q(H(L)) \neq \emptyset,\left(\partial E_{n}\right) \cap q(H(L))=\emptyset$, and $\left(E_{n+1}-E_{n}\right) \cap q(H(L)) \neq \emptyset$ for all $n \geq 0$. Choose a properly embedded arc $\alpha_{n+1}$ in $E_{n+1}-E_{n}$ which splits $E_{n+1}-$ int $E_{n}$ into a disk $\mathcal{E}_{n+1}$ and an annulus $A_{n+1}$ such that $A_{n+1} \cap q(H(L))=$ $\emptyset$.

We define $B_{n}, \mathcal{D}_{n+1}, \mathcal{B}_{n+1}$, and $\Sigma_{n+1}$ as follows.

$$
\begin{aligned}
B_{n} & =H^{-1}\left(E_{n} \times[-(n+1), n+1]\right) \\
\mathcal{D}_{n+1} & =H^{-1}\left(\alpha_{n+1} \times[-(n+2), n+2]\right) \\
\mathcal{B}_{n+1} & =H^{-1}\left(\mathcal{E}_{n+1} \times[-(n+2), n+2]\right) \\
\Sigma_{n+1} & =B_{n+1}-\left(\operatorname{int} B_{n} \cup \operatorname{Int} \mathcal{B}_{n+1}\right)
\end{aligned}
$$


Then the exhaustion $\left\{B_{n}\right\}_{n \geq 0}$ of $\mathbb{R}^{3}$ has the required properties. In particular the existence of $\mathcal{D}_{n+1}, \Sigma_{n+1}$, and $\mathcal{B}_{n+1}$ establishes (3).

Now suppose that $\left\{B_{n}\right\}_{n \geq 0}$ is an exhaustion satisfying the three properties.

We construct $H$ inductively. Let $\left\{E_{n}\right\}_{n \geq 0}$ be an exhaustion of $\mathbb{R}^{2}$ by disks.

Since $B_{0} \cap L$ is a trivial $k_{0}$-tangle there is a homeomorphism $H_{0}:\left(B_{0}, B_{0} \cap L\right) \rightarrow$ $\left(E_{0}, P_{0}\right) \times[-1,1]$, where $P_{0}$ is a set of $k_{0}$ points in int $E_{0}$.

Suppose we have constructed a homeomorphism

$$
H_{n}:\left(B_{n}, B_{n} \cap L\right) \rightarrow\left(E_{n}, P_{n}\right) \times[-(n+1), n+1],
$$

where $P_{n}$ is a set of $k_{n}$ points in int $E_{n}$.

Choose a properly embedded $\operatorname{arc} \alpha_{n+1}$ in $E_{n+1}-E_{n}$. Then $\alpha_{n+1}$ splits $E_{n+1}-$ int $E_{n}$ into an annulus $A_{n+1}$ and a disk $\mathcal{E}_{n+1}$. Suppose $B_{n+1} \cap L$ has $k_{n+1}$ components. Let $P_{n+1}$ be the union of $P_{n}$ and a set of $k_{n+1}-k_{n}$ points in $\operatorname{int} \mathcal{E}_{n+1}$.

By (3) and Lemma 7.3 (1) there is a properly embedded disk $\mathcal{D}_{n+1}$ in $B_{n+1}-B_{n}$ which splits $B_{n+1}-\operatorname{int} B_{n}$ into a shell $\Sigma_{n+1}$ and a 3 -ball $\mathcal{B}_{n+1}$ such that $\left(\Sigma_{n+1}, \Sigma_{n+1} \cap L\right)$ is a $2 k_{n}$-braid and $\left(\mathcal{B}_{n+1}, \mathcal{B}_{n+1} \cap L\right)$ is a trivial $\left(k_{n+1}-k_{n}\right)$ tangle.

We define a shell $S_{n+1}$ as follows.

$$
\left.S_{n+1}=\left(\left(E_{n} \cup A_{n+1}\right) \times[-(n+2), n+2)\right]\right)-\operatorname{int}\left(E_{n} \times[-(n+1), n+1]\right)
$$

The restriction of $H_{n}$ to $\left(\partial B_{n}, \partial B_{n} \cap L\right)$ extends to a homeomorphism

$$
H_{n+1}^{\Sigma}:\left(\Sigma_{n+1}, \Sigma_{n+1} \cap L\right) \rightarrow\left(S_{n+1}, S_{n+1} \cap\left(P_{n} \times[-(n+2), n+2]\right)\right) .
$$

The restriction of $H_{n+1}^{\Sigma}$ to

$$
\Sigma_{n+1} \cap \mathcal{B}_{n+1}=\left(H_{n+1}^{\Sigma}\right)^{-1}\left(\alpha_{n+1} \times[-(n+2), n+2]\right)
$$

then extends to a homeomorphism

$$
H_{n+1}^{\mathcal{B}}:\left(\mathcal{B}_{n+1}, \mathcal{B}_{n+1} \cap L\right) \rightarrow\left(\mathcal{E}_{n+1}, P_{n+1}-P_{n}\right) \times[-(n+2), n+2] .
$$

We then use $H_{n}, H_{n+1}^{\Sigma}$, and $H_{n+1}^{\mathcal{B}}$ to define

$$
H_{n+1}:\left(B_{n+1}, B_{n+1} \cap L\right) \rightarrow\left(E_{n+1}, P_{n+1}\right) \times[-(n+2), n+2] .
$$

Proof of Theorem 8.1 Given an exhaustion $\left\{B_{n}\right\}$ for $\widetilde{M}$ which satisfies the conditions of Lemma 8.2, we will construct an exhaustion $\left\{B_{n}^{*}\right\}$ for $\widetilde{V}$ which satisfies these conditions. We may assume that $B_{0} \subseteq \widetilde{V}$. Let $B_{0}^{*}=B_{0}$. 
The proof follows that of Theorem 6.1. We assume that $V$ is the end reduction obtained from an exhaustion $\left\{C_{n}\right\}$ for $M$ by cutting 1-handles.

Suppose $A_{0}$ is a compact, connected subset of $\widetilde{V}$. We may assume that the interior of $B_{1}$ contains $A_{0} \cup B_{0}^{*}$ and that $\left(p\left(B_{1}\right), p\left(A_{0} \cup B_{0}^{*}\right)\right) \subseteq\left(C_{1}, C_{1}^{*} \cap V\right)$.

Let $D$ be the co-core of the first 1 -handle of $C_{1}$ which is cut. Then $D$ compresses $\partial C_{1}$ in $C_{1}-\gamma$. Let $C_{1}^{\prime}$ be the resulting $3-$ manifold. Then $p^{-1}(D)$ is a disjoint union of disks $\widetilde{D}_{i}$ in $p^{-1}\left(C_{1}\right)$. Only finitely many $\widetilde{D}_{i}$ meet $B_{1}$. None of them meet $A_{0} \cup B_{0}^{*} \cup p^{-1}(\gamma)$. Put $\partial B_{1}$ in general position with respect to $p^{-1}(D)$. Assume that for some $i$ we have $\widetilde{D}_{i} \cap B \neq \emptyset$. Let $\Delta$ be an innermost disk on $\widetilde{D}_{i}$, ie $\Delta \cap \partial B=\partial \Delta$.

Suppose $\Delta$ lies in $B_{1}$. Then it splits $B_{1}$ into two 3 -balls one of which, call it $B^{\#}$, contains $A_{0} \cup B_{0}^{*}$. Let $\tau^{\#}=L \cap B^{\#}$. Then by Lemma $7.4\left(B_{0}^{*}, B_{0}^{*} \cap L\right)$ is standardly embedded in $\left(B^{\#}, B^{\#} \cap L\right)$, and by Lemmas $7.3(4)$ and 7.5 $\left(B^{\#}, B^{\#} \cap L\right)$ is standardly embedded in $\left(B_{n}, B_{n} \cap L\right)$ for all $n \geq 1$.

Now suppose that $\Delta$ lies in $\widetilde{M}-\operatorname{int} B_{1}$. Attaching a 2 -handle to $B_{1}$ with core $\Delta$ gives a 3 -manifold $Q$ with $\partial Q$ a disjoint union of two $2-$ spheres. One of them bounds a 3 -ball $B^{\#}$ in $\widetilde{M}$ which contains $Q . B^{\#}$ is the union of $B_{1}$ and a 3 -ball $B^{+}$such that $B^{\#} \cap B^{+}$is a disk. Since by Lemma 7.3 (4) $\left(B_{1}, B_{1} \cap L\right)$ is standardly embedded in $\left(B_{m^{\#}}, B_{m^{\#}} \cap L\right)$ for some $m^{\#} \geq 1$ such that $B^{\#} \subseteq \operatorname{int} B_{m \#}$ we have that $B^{+} \cap L=\emptyset$. It follows that $\left(B_{0}^{*}, B_{0}^{*} \cap L\right)$ is standardly embedded in $\left(B^{\#}, B^{\#} \cap L\right)$ which by Lemma 7.3 (4) is itself standardly embedded in $\left(B_{n}, B_{n} \cap L\right)$ for all $n \geq m^{\#}$.

Note that in both cases we may isotop $B^{\#}$ slightly so that $\partial B^{\#}$ has at least one fewer intersection curve with $p^{-1}(D)$ than did $\partial B_{1}$. Continuing in this manner we get a 3 -ball $B^{\prime}$ in $\widetilde{M}$ such that $B^{\prime} \cap p^{-1}(D)=\emptyset,\left(A_{0} \cup B_{0}^{*}\right) \subseteq \operatorname{int} B^{\prime}$, $B^{\prime} \subseteq \operatorname{int} p^{-1}\left(C_{1}^{\prime}\right),\left(B_{0}^{*}, B_{0}^{*} \cap L\right)$ is standardly embedded in $\left(B^{\prime}, B^{\prime} \cap L\right)$, and for some $m^{\prime} \geq 1$ we have that $\left(B^{\prime}, B^{\prime} \cap L\right)$ is standardly embedded in $\left(B_{n}, B_{n} \cap L\right)$ for all $n \geq m^{\prime}$.

We then cut the next 1 -handle. We eventually get a 3 -ball $B_{1}^{*}$ in $\widetilde{M}$ such that $\left(A_{0} \cup B_{0}^{*}\right) \subseteq \operatorname{int} B_{1}^{*}, B_{1}^{*} \subseteq p^{-1}\left(C_{1}^{*} \cap V\right) \cap \widetilde{V},\left(B_{0}^{*}, B_{0}^{*} \cap L\right)$ is standardly embedded in $\left(B_{1}^{*}, B_{1}^{*} \cap L\right)$, and for some $m^{*} \geq 1$ we have that $\left(B_{1}^{*}, B_{1}^{*} \cap L\right)$ is standardly embedded in $\left(B_{n}, B_{n} \cap L\right)$ for all $n \geq m^{*}$.

We then repeat this argument inductively to construct the exhaustion $\left\{B_{n}^{*}\right\}$. 


\section{End reductions at links}

In this section we consider end reductions at non-connected subsets of $M$. Let $J$ be a compact but not necessarily connected $3-$ manifold in $M$. As before we define $J$ to be regular if $M-J$ is irreducible and has no component with compact closure. End irreducibility and the engulfing property rel $J$ in $M$ and end reductions of $M$ at $J$ are defined as before. The standard end reduction and uniqueness up to non-ambient isotopy work as before. However, $V$ may not be connected, and a component of $V^{\prime}$ of $V$ may not be an end reduction at $V^{\prime} \cap J$. As before, each component of $V$ is $\pi_{1}$-injective in $M$.

A regular link in $M$ is a disjoint union $\gamma$ of finitely many simple closed curves in $M$ such that $M-\gamma$ is irreducible. An end reduction of $M$ at $\gamma$ is defined to be an end reduction of $M$ at a regular neighborhood of $\gamma$.

Note that if each component of a link $\gamma$ is homotopically non-trivial in $M$, then $\gamma$ is a regular link.

Theorem 9.1 (Agol) Let $M$ be a connected, orientable, irreducible open 3manifold. Let $\gamma$ be a link in $M$ such that no component of $\gamma$ is homotopically trivial in $M$. Let $V$ be an end reduction of $M$ at $\gamma$. Suppose $\pi_{1}(M)$ is finitely generated and decomposable, and that some component of $\gamma$ is algebraically disk busting. Then $V$ is connected and $\pi_{1}(V) \rightarrow \pi_{1}(M)$ is an isomorphism.

We will prove a slightly more general statement. Let $\gamma=\gamma_{1} \cup \cdots \cup \gamma_{m}$ be a link in $M$ such that no component of $\gamma$ is homotopically trivial in $M$. It is algebraically disk busting if there is no proper free factorization $A_{1} * A_{2}$ of $\pi_{1}(M)$ such that for each $i$ there is a $j$ such that $\gamma_{i}$ is conjugate into $A_{j}$. In general having an algebraically disk busting component is sufficient, but not necessary, for $\gamma$ to be algebraically disk busting. If $\pi_{1}(M)$ is indecomposable, then $\gamma$ is automatically algebraically disk busting.

Theorem 9.2 Let $M$ be a connected, orientable, irreducible open 3-manifold. Let $\gamma$ be a link in $M$ no component of which is homotopically trivial in $M$. Let $V$ be an end reduction of $M$ at $\gamma$. Suppose $\pi_{1}(M)$ is finitely generated and $\gamma$ is algebraically disk busting. Then $V$ is connected and $\pi_{1}(V) \rightarrow \pi_{1}(M)$ is an isomorphism.

Proof First suppose that $\pi_{1}(M)$ is indecomposable. As in the proof of Theorem 4.1 we isotop a compact core $N$ so that it lies in $V^{*}$. Suppose there are distinct components $U$ and $U^{\prime}$ of $V^{*}$ such that $U$ is a component of $V$ and 
$U^{\prime}$ contains $N$. Then $\pi_{1}(U)$ is non-trivial. However, as before, a homotopy between a loop in $U$ and a loop in $N$ could be cut off on the co-core of a 1 -handle that is cut in the process of constructing $V^{*}$. Thus $N$ lies in $V$, and $V$ is connected. Hence $\pi_{1}(V) \rightarrow \pi_{1}(M)$ is an isomorphism.

Now suppose that $\pi_{1}(M)$ is decomposable. As in the proof of Theorem 5.1 we assume that a compact core $N$ is contained in $\operatorname{int} C_{1}$ and that the link $\gamma$ is freely homotopic in int $C_{1}$ to a link $\gamma^{\prime}$ in $\operatorname{int} N$. We again let $D$ be the co-core of the first 1-handle which is cut from $C_{1}$ and may assume that $N \cap D$ consists of a minimal number of essential disks in $N$.

We may assume that $\gamma^{\prime} \cap D$ has a minimal number of points. If $\gamma^{\prime} \cap D=\emptyset$, then some essential disk in $N$ misses $\gamma^{\prime}$, hence the components of $\gamma^{\prime}$ lie in components of $N$ split along this disk, and so the components of $\gamma$ are conjugate into factors of a proper free factorization of $\pi_{1}(M)$. This contradicts the fact that $\gamma$ is algebraically disk busting. Thus $\gamma^{\prime} \cap D \neq \emptyset$.

Let $S$ be a disjoint union of $m$ copies $S_{i}^{1}$ of $S^{1}$. We have a map $f: S \times[0,1] \rightarrow$ $C_{1}$ with $f(S \times\{0\})=\gamma$ and $f(S \times\{1\})=\gamma^{\prime}$. The argument now proceeds exactly as in Theorem 5.1 to contradict either the minimality of $\gamma^{\prime} \cap D$ or that of $N \cap D$.

Thus $N \cap D=\emptyset$. We repeat the argument to get that $N \subseteq C_{1}^{*}$. As in the indecomposable case we get that $V$ is connected and $N \subseteq V$.

\section{References}

[1] I Agol, Tameness of hyperbolic 3-manifolds, arXiv:math.GT/0405568

[2] MG Brin, TL Thickstun, Open, irreducible 3-manifolds which are end 1movable, Topology 26 (1987) 211-233 MathReview

[3] M G Brin, TL Thickstun, 3-manifolds which are end 1-movable, Memoirs Amer. Math. Soc. 81 (1989) MathReview

[4] EM Brown, CD Feustel, On properly embedding planes in arbitrary 3manifolds, Proc. Amer. Math. Soc. 94 (1985) 173-178 MathReview

[5] D C Calegari, D Gabai, Shrinkwrapping and the taming of hyperbolic 3manifolds, arXiv:math.GT/0407161

[6] A V Chernavskii, Local contractibility of the group of homeomorphisms of a manifold, Mat. Sb. (N.S.) 79 (121) (1969) 307-356 MathReview

[7] R D Edwards, R C Kirby, Deformations of spaces of imbeddings, Ann. Math. (2) 93 (1971) 63-88 MathReview 
[8] M Freedman, D Gabai, Covering a nontaming knot by the unlink, preprint available at http://research.microsoft.com/theory/freedman/or at http://www.math.princeton.edu/facultypapers/Gabai/

[9] M Freedman, V Krushkal, Notes on ends of hyperbolic 3-manifolds, from: "Proc. 13th Annual Workshop in Geometric Topology, Colorado College, Colorado Springs, CO (June 13-15, 1996)" 1-15

[10] H Freudenthal, Neuaufbau der Endentheorie, Ann. of Math. (2) 43 (1942) 261-279 MathReview

[11] J Hempel, 3-manifolds, Ann. of Math. Studies 86, Princeton (1976) MathReview

[12] W Jaco, Lectures on three-manifold topology, CBMS Regional Conference Series in Math. 43, Amer. Math. Soc. (1980) MathReview

[13] D R McMillan Jr, Cartesian products of contractible open manifolds, Bull. Amer. Math. Soc. 67 (1961) 510-514 MathReview

[14] D R McMillan Jr, Compact, acyclic subsets of three-manifolds, Michigan Math. J. 16 (1969) 129-136 MathReview

[15] D R McMillan Jr, Acyclicity in three-manifolds, Bull. Amer. Math. Soc. 76 (1970) 942-964 MathReview

[16] R Myers, End reductions and covering translations of contractible open 3manifolds, arXiv:math.GT/0209397

[17] P Scott, Compact submanifolds of 3-manifolds, J. London Math. Soc. (2) 7 (1973) 246-250 MathReview 